\title{
Atitudes e Representações em Saúde Mental: Um Estudo com Universitários
}

\author{
Patrícia Fonseca de Sousa - Universidade Federal da Paraíba, João Pessoa, Brasil \\ Silvana Carneiro Maciel - Universidade Federal da Paraíba, João Pessoa, Brasil \\ Katruccy Tenório Medeiros - Universidade Federal da Paraíba, João Pessoa, Brasil \\ Giselli Lucy Sonza Vieira - Universidade Federal da Paraíba, João Pessoa, Brasil
}

\begin{abstract}
Resumo
Este estudo teve como objetivo conhecer e analisar as representações sociais de universitários sobre a Reforma Psiquiátrica e o doente mental, relacionando-as com a adesão destes aos paradigmas de atenção à saúde mental. Participaram 480 universitários dos cursos de Psicologia, Medicina e Enfermagem; a maioria do sexo feminino (74,4\%) e com idade média de 24 anos $(D P=$ 5,98). Os dados foram coletados utilizando-se Escala de Atitudes em Saúde Mental e técnica de Associação Livre de Palavras. Os resultados apontaram uma compreensão ambígua acerca da Reforma Psiquiátrica. Os universitários demonstraram conhecer as práticas desse novo modelo e apresentaram maior adesão ao paradigma psicossocial, mas observou-se uma representação do doente mental marcada pelo medo e exclusão e vinculada ao paradigma biomédico. Tal achado parece ser um reflexo da atual situação do campo da saúde mental, na qual o paradigma biomédico não foi totalmente superado e nem o paradigma psicossocial totalmente estabelecido.
\end{abstract}

Palavras-chave: reforma psiquiátrica, estudantes universitários, representação social

\section{Attitudes and Representation in Mental Health: A Study with University Students}

\begin{abstract}
This study aims to know and analyze university students' social representations on the psychiatric reform and on mentally ill people, relating them to their adhesion to attention to mental health paradigms. 480 Psychology, Medicine and Nursing university students participated in this research, most of them females $(74.4 \%)$ and with average age of 24 years $(S D=5.98)$. Data were collected through the Scale of Attitudes in Mental Health and the Technique of Free Association of Words. Results revealed ambiguous comprehension of the psychiatric reform. University students demonstrated to know practices of this new model and presented higher adhesion to psychosocial paradigms, but a representation of the mentally ill person marked by fear and exclusion and linked to biomedical paradigm was observed. Such finding seems to reflect the present situation in the mental health field, in which the biomedical paradigm was not totally overcome nor the psychosocial paradigm totally established. Keywords: psychiatric reform, college students, social representation
\end{abstract}

\section{Actitudes y Representaciones en Salud Mental: Un Estudio con Estudiantes Universitarios}

\section{Resumen}

El objetivo de este estudio fue conocer y analizar las representaciones sociales de universitarios sobre la Reforma Psiquiátrica y el enfermo mental, relacionándolas con su adhesión a la atención de los paradigmas de salud mental. Participaron 480 estudiantes universitarios de los cursos de Psicología, Medicina y Enfermería; la mayoría de sexo femenino (74,4\%), con edad media de 24 años $(D P=5,98)$. Los datos fueron recogidos utilizando la Escala de Actitudes en Salud Mental y Técnica de Asociación Libre de Palabras. Los resultados señalaron comprensión ambigua sobre la reforma psiquiátrica. Los estudiantes demostraron conocer las prácticas del nuevo modelo y presentaron mayor adhesión al paradigma psicosocial, pero se observó una representación del enfermo mental marcada por el miedo y la exclusión vinculada al paradigma biomédico. Este resultado parece ser un reflejo de la situación actual en el área de salud mental, donde el paradigma biomédico no fue plenamente superado y el paradigma psicosocial no fue totalmente establecido.

Palabras-clave: reforma psiquiátrica, estudiantes universitarios, representación social

Tradicionalmente, a sociedade percebe o fenômeno da doença mental como relacionado à improdutividade, a algo negativo, suscetível de reclusão e, sobretudo, à periculosidade. Tal percepção contribuiu para a disseminação de atitudes negativas como o preconceito e para a dificuldade de inserção social das pessoas com sofrimento psíquico, configurando práticas no âmbito da assistência à saúde mental que, historicamente, foram marcadas pela rotulação, supressão dos sintomas por meio da medicalização e pelo confinamento em instituições especializadas (Maciel, Barros, Camino, \& Melo, 2011). Porém, nas últimas décadas, com o advento da reforma psiquiátrica, aconteceram mudanças no cenário da saúde mental, no Brasil e no mundo, que contribuíram para uma assistência mais humanizada ao portador de sofrimento psíquico (Barros \& Claro, 2011; Menegat, 2010). 
A Reforma Psiquiátrica surgiu no sentido de modificar o sistema de tratamento da doença mental, buscando alternativas substitutivas ao modelo hospitalocêntrico. Buscando, assim, a eliminação gradual da internação, entendida como mecanismo de exclusão social e substituindo-a por uma rede de serviços territoriais de atenção psicossocial, com o objetivo de integrar o portador de transtorno mental ao convívio social (Calgaro \& Souza, 2009).

No Brasil, os primeiros delineamentos da Reforma Psiquiátrica tiveram início na segunda metade da década de 1970, com o surgimento de uma série de denúncias acerca da péssima qualidade dos serviços e da falta de assistência médica adequada nas instituições psiquiátricas. Um importante marco da Reforma Psiquiátrica foi a aprovação da Lei $\mathrm{n}^{\circ} 10.216$ ou Lei da Reforma Psiquiátrica Brasileira, em 2001; essa lei oficializou o atendimento psiquiátrico comunitário no Brasil, dispôs sobre o tratamento mais humanizado, a proteção às pessoas com transtornos psiquiátricos, a preferência pelos serviços comunitários, a implantação, em todo o território nacional, de serviços substitutivos e a regulamentação das internações compulsórias (Guimarães et al., 2010).

A Reforma Psiquiátrica propõe uma transição paradigmática, estabelecendo um modelo de atenção psicossocial em substituição ao modelo biomédico. $\mathrm{O}$ modelo biomédico institui o hospital psiquiátrico como o local de escolha para o tratamento, caracteriza-se pelo trabalho centrado na figura do médico e pela ênfase nas determinações orgânicas dos problemas (doenças) e na terapêutica medicamentosa; propiciando a exclusão de familiares e de usuários no processo de tratamento. Em contraposição, o modelo psicossocial, advindo com a Reforma Psiquiátrica, caracteriza-se pelo trabalho em equipe multidisciplinar e uso de diferentes recursos terapêuticos; enfatizando a reinserção social do indivíduo, investindo no trabalho com a família e com a comunidade e incentivando o uso de dispositivos extra-hospitalares (Costa-Rosa, 2012; Maciel, 2007).

Contudo, apesar dos avanços no âmbito da saúde mental, inúmeros desafios ainda se apresentam no cenário da Reforma Psiquiátrica, afetando a sua sustentabilidade. Dentre eles, pode-se citar a dificuldade de reinserção social dos portadores de transtorno mental, devido às representações negativas que ainda persistem na sociedade acerca de tais indivíduos (Maciel, 2007); mesmo entre os profissionais da saúde mental. Acerca disso, Paes, Maftum e Mantovani (2010) em estudo com 64 profissionais de Enfermagem sobre o atendimento ao paciente com comorbidade psiquiátrica, em um Hospital Geral da cidade de Curitiba, observaram que esses profissionais apresentavam sentimentos de medo de agressão e de insegurança diante dos portadores de transtornos mentais; percebendo-os de forma preconceituosa, e acarretando atitudes de exclusão e uma prática pouco reabilitativa por parte desses profissionais.

Observa-se, pois, que o processo de exclusão social dos portadores de transtornos mentais, não é algo atual nem pontual, mas perpassa séculos de construção de representações estereotipadas, revelando o claro despreparo da população para o convívio com o doente mental, observado por meio das atitudes de exclusão ao longo da história (Foucault, 2012; Liberato, 2009). Diante disso, faz-se importante o resgate e a articulação de dois conceitos para a maior compreensão do processo de exclusão social do doente mental: representações e atitudes. Segundo Fagundes, Zanella e Torres (2012), a articulação entre tais conceitos é de grande relevância por estar intimamente vinculada à prática apresentada pelas pessoas, permitindo, dessa forma, compreender como os sujeitos se comportam e como justificam ou se posicionam em relação às suas ações.

De acordo com Lima (2006), a atitude é um dos mais antigos e estudados conceitos em Psicologia Social e recebeu diversas definições em distintas épocas. Conforme esse autor, atitude pode ser entendida como uma organização duradoura de crenças e cognições em geral, dotada de carga afetiva pró ou contra um objeto social definido, que predispõe a uma ação coerente com cognições e afetos relativos a esse objeto. A partir de tal conceito, pode-se afirmar que as atitudes possuem três componentes: cognitivo, afetivo (pró ou contra) e uma predisposição comportamental.

Quanto às representações sociais, Saraiva e Coutinho (2007) afirmam que estas são conjuntos simbólicos/ práticos/dinâmicos cujo status é o de uma produção e não de uma reprodução, ou seja, constituem não uma simples reação a estímulos exteriores, mas sim a seleção e utilização, a partir do repertório circundante na sociedade, de informações destinadas à interpretação e à elaboração do real. Dessa forma, a representação social de estudantes universitários sobre a doente mental e Reforma Psiquiátrica pode ser compreendida como uma interpretação da realidade vivida e falada por esse grupo social, direcionando comportamentos e comunicações (Vieira, Saraiva, \& Coutinho, 2010).

Ainda em relação às representações sociais, Moscovici (1961) destaca que estas são compostas pela 
informação (qualidade e quantidade de conhecimento que o sujeito/grupo tem sobre o objeto), pelas atitudes (possibilidade de identificar posições favoráveis ou não em relação a determinado objeto) e pelo campo de representação (que permite a visualização de conhecimentos e atitudes organizados em forma de teoria).

Nesse contexto, vale destacar que, para instrumentalizar este estudo, utilizou-se a abordagem estrutural das representações sociais, Teoria do Núcleo Central (Abric, 2003), esta corresponde a uma abordagem complementar ao estudo das representações sociais empreendido por Moscovici, de modo a torná-lo mais heurístico, tanto na prática social como na pesquisa. O modelo proposto por Abric caracteriza-se por considerar que as representações sociais são estruturadas em um núcleo central e em elementos periféricos (Sá, 1996).

Conforme Abric (2003), o núcleo central caracteriza-se como estável e rígido, por ser marcado e determinado pelas condições históricas, sociológicas e ideológicas, estando, dessa forma, fortemente enraizado na memória coletiva de um grupo e balizado pelo seu sistema de normas e valores; o núcleo é determinado pela natureza do objeto representado e pelo tipo de relação mantida entre grupo e objeto. Já os elementos periféricos caracterizam-se como móveis e flexíveis, por se abastecerem das experiências individuais, integrando os dados do vivido e da situação específica, seus conteúdos são vivos, concretos e de fácil acesso.

De acordo com Jodelet (2001), as representações sociais desempenham um papel importante na vida das pessoas, na medida em que funcionam como guias no modo de nomear e definir os diferentes aspectos da realidade diária, no modo de interpretar esses aspectos, tomar decisões e posicionar-se frente a eles. Assim, as representações sociais elaboradas por estudantes universitários acerca do doente mental e da Reforma Psiquiátrica são valiosos instrumentos para se compreender onde estarão pautadas as práticas desses futuros profissionais, que atuarão na área da saúde mental, tendo em vista o novo modelo de assistência à saúde mental, preconizada pela Reforma Psiquiátrica.

Cabe ressaltar que o conhecimento do conteúdo de uma representação não é suficiente para defini-la, sendo necessário identificar o seu núcleo central, pois é ele que possibilita à representação sua significação, determina os laços que unem entre si os elementos do conteúdo e que regem sua evolução e transformação (Abric, 2003). Nesse sentido, Sá (1996) assinala que duas representações podem possuir o mesmo conteúdo, mas ter significações diferentes, em virtude da sua estrutura. Psico-USF, Bragança Paulista, v. 21, n. 3, p. 527-538, set./ dez. 2016
Diante disso, o presente estudo teve como objetivo conhecer e analisar as representações sociais de estudantes dos cursos de Psicologia, Medicina e Enfermagem sobre a Reforma Psiquiátrica e o doente mental, relacionando-as com a sua adesão aos paradigmas de atenção à saúde mental (atitude frente à Reforma Psiquiátrica). $\mathrm{O}$ intuito foi o de compreender como esses futuros profissionais do campo da saúde mental representam tais objetos sociais e se posicionam diante deles, diante da nova configuração do campo da saúde mental, uma vez que, no futuro, estarão inseridos nos serviços substitutivos e tais representações e atitudes poderão repercutir na sua atuação profissional.

\section{Método}

\section{Participantes}

Participaram desta pesquisa 480 estudantes universitários, distribuídos entre os cursos de Psicologia, Medicina e Enfermagem, de Universidades públicas e privadas localizadas na cidade de (informação retirada para preservar o anonimato do autor), sendo 160 estudantes de cada curso. Destes, 25,6\% pertenciam ao sexo masculino e $74,4 \%$ pertenciam ao sexo feminino e apresentavam idade média de 24 anos $(D P=5,98)$. Optou-se por tais cursos pelo fato de estarem vinculados ao trabalho na rede de saúde mental, tratando-se de uma amostra de conveniência (não probabilística).

\section{Instrumentos}

Para a coleta de dados, foram utilizadas a Escala de Atitudes em Saúde Mental (Sousa et al., no prelo) e a Técnica de Associação Livre de Palavras (TALP).

A Escala de Atitudes em Saúde Mental (EASM) busca avaliar o posicionamento dos indivíduos frente aos paradigmas biomédico e psicossocial de atenção à saúde mental, é composta por 15 itens, distribuídos em duas subescalas; (a) paradigma biomédico (Ex. item: $\mathrm{O}$ doente mental deve ser isolado quando fica agressivo fisicamente; alfa de Cronbach: 0,71); e (b) paradigma psicossocial (Ex. item: Os vínculos sociais podem tratar um paciente psiquiátrico; alfa de Cronbach: 0,66). Os itens dessa escala estão organizados em formato Likert, variando de 1 (Discorda plenamente) a 7 (Concorda plenamente).

A TALP é uma técnica usada nas pesquisas sobre as representações sociais, que consiste em solicitar aos participantes que digam as palavras ou expressões que lhes venham imediatamente à lembrança, quando um estímulo indutor for apresentado pelo pesquisador 
(Nóbrega \& Coutinho, 2011). A TALP foi utilizada com os seguintes estímulos indutores: "reforma psiquiátrica" e "doente mental". Cabe salientar que se optou por usar o termo "doente mental" pelo fato de este, ainda, ser comumente usado pela população geral e por profissionais da saúde mental para referir-se às pessoas que apresentam algum sofrimento psíquico. Por fim, os participantes responderam a um questionário sociodemográfico (idade, sexo, curso, período, instituição onde estuda), para a caracterização da amostra.

\section{Procedimento}

Após a obtenção da autorização para a realização da pesquisa, pelo Comitê de Ética XXXXX, contataram-se as instituições onde foi realizado o estudo. A partir do consentimento dos coordenadores destas, iniciou-se a coleta. Primeiramente, foram fornecidas informações aos participantes sobre o objetivo do estudo, a inexistência de respostas certas ou erradas, o direito de optar por participar ou não e a garantia de anonimato. Após a comunicação dessas questões e mediante a aceitação dos estudantes em participar, obtida por meio da assinatura do Termo de Consentimento Livre e Esclarecido, iniciou-se a aplicação dos instrumentos, de forma coletiva, nas salas de aula, em horário previamente combinado com os professores. Os aplicadores foram estudantes universitários treinados para a realização de tal procedimento. Aplicou-se inicialmente a TALP, precedida de explicações gerais; em seguida, foi aplicada a Escala de Atitudes em Saúde Mental (EASM) e, por fim, o questionário sociodemográfico. Os participantes levaram, em média, 15 minutos para concluir a participação na pesquisa.

\section{Análise dos Dados}

Os dados da escala e do questionário sociodemográfico foram tabulados e analisados por meio do Pacote Estatístico para Ciências Sociais (SPSS) para Windows, versão 21.0. $\mathrm{Na}$ análise da escala, além de procedimentos estatísticos descritivos (média, desvio padrão, etc.), também foram realizadas análises de variância (ANOVA) e testes t. $\mathrm{Na}$ análise do questionário sociodemográfico, utilizaram-se frequências e porcentagens (estatística descritiva). As distribuições das pontuações das variáveis utilizadas foram testadas por meio do teste de Kolmogorov-Smirnov, para verificar se estas eram significativamente diferentes da normalidade. Como não foram encontrados desvios significativos da normalidade, adotaram-se testes estatísticos paramétricos. Também foi verificada a hipótese de homogeneidade de variâncias, por meio do teste de Levene, o qual indicou que tal hipótese não foi violada (Field, 2009).

Os dados obtidos por meio da TALP foram processados com a utilização do programa computacional EVOC (Ensemble de programmes permettant l'analyse des evocations). Por meio da análise lexicográfica, esse programa permite demonstrar graficamente as palavras pertencentes ao núcleo central e ao sistema periférico das representações sociais, identificando a frequência de aparecimento dos termos evocados e a ordem de aparecimento das respostas registradas (Saraiva, Vieira, \& Coutinho, 2011).

\section{Resultados e Discussões}

\section{Escala de Atitudes em Saúde Mental}

Visando demonstrar a adesão dos universitários aos paradigmas de atenção à saúde mental, aplicou-se a Escala de Atitudes em Saúde Mental (composta pelos fatores paradigma psicossocial e paradigma biomédico). Vale acrescentar que altos escores em um dos fatores são interpretados como indicadores de adesão ao paradigma biomédico ou psicossocial. Os resultados da Escala são mostrados na Tabela 1:

Como pode ser observado na Tabela 1, os universitários dos três cursos apresentaram uma maior

Tabela 1

Média e Desvio Padrão de Universitários na Escala de Atitudes em Saúde Mental

\begin{tabular}{lcccc}
\hline \multirow{2}{*}{ Cursos } & \multicolumn{2}{c}{ Paradigma biomédico } & \multicolumn{2}{c}{ Paradigma psicossocial } \\
\cline { 2 - 5 } & $\mathrm{M}$ & $\mathrm{DP}$ & $\mathrm{M}$ & $\mathrm{DP}$ \\
\hline Psicologia & 18,41 & 6,82 & 33,10 & 6,84 \\
Medicina & 24,32 & 7,06 & 31,18 & 5,15 \\
Enfermagem & 21,52 & 6,84 & 30,80 & 5,81 \\
\hline
\end{tabular}


pontuação no paradigma psicossocial (Psicologia: $t$ $(158)=-16,46, p<0,05$; Medicina: $t(159)=-8,70, p<$ $0,05$; Enfermagem: $t(158)=-11,02, p<0,05)$. O paradigma psicossocial compreende que as perspectivas de atenção estão voltadas para um investimento na maior autonomia e independência da pessoa com transtorno mental, buscando-se uma ressocialização e reabilitação psicossocial desses indivíduos para que aconteça uma efetiva inclusão social (Costa-Rosa, 2012).

Tais dados podem ser atribuídos aos avanços ao longo dos trinta anos de Reforma Psiquiátrica, apesar dos inúmeros desafios ainda existentes. Sobre os avanços, Parente, Menezes, Branco, Sales e Parente (2013) mencionam o aumento no número de publicações científicas na área da exclusão social, a ampliação da contratação de profissionais para atuarem no campo da saúde mental, a expansão da rede de serviços substitutivos e as ações voltadas para a formação permanente de profissionais. Para tais autores, essas mudanças fizeram com que a Reforma Psiquiátrica ganhasse visibilidade na sociedade e suas propostas começassem a ser assimiladas pela população.

Mas, embora os estudantes tenham apresentado maior pontuação no paradigma psicossocial, buscou-se verificar qual curso apresentava maior pontuação no paradigma biomédico e qual apresentava maior pontuação no paradigma psicossocial.

Uma ANOVA foi executada e indicou que há diferença entre as médias dos universitários no paradigma biomédico $(\mathrm{F}(2,477)=29,31, p<0,001)$, e o teste de Bonferroni esclareceu onde ocorreram as diferenças. Assim, observou-se que há diferenças entre os cursos de Psicologia e de Medicina $(p<0,001)$; de Psicologia e de Enfermagem $(p<0,001)$ e de Medicina e Enfermagem $(p<0,001)$; assim, o curso que mais pontuou no paradigma biomédico foi o de Medicina.

Tal achado pode ser atribuído ao fato de tradicionalmente os cursos de Medicina concentrarem sua ênfase na doença e não no indivíduo; o ensino ser essencialmente hospitalocêntrico (pouca ênfase em outros cenários de prática, como, por exemplo, a comunidade); não se ressaltar, durante a formação dos médicos, o papel social destes e haver ainda a redução de profissionais que apresentam uma visão holística do indivíduo, devido ao predomínio das especializações, conforme afirmam Gonçalves e Benevides-Pereira (2009).

Nessa perspectiva, uma pesquisa realizada por Pagliosa (2006), com estudantes de Medicina da Universidade Federal de Santa Catarina, mostrou que esses universitários possuíam um entendimento do processo saúde-doença caracterizado por uma forte conotação biológica. A compreensão do funcionamento do corpo apareceu como uma das principais preocupações, com destaque para o estudo da anatomia. Além disso, verificou-se que as disciplinas que não apresentavam caráter biológico, como Antropologia e Sociologia, foram consideradas como de pouca importância por esses estudantes.

Diante das mudanças no campo da saúde, alterações foram implementadas na graduação em Medicina, enfatizando-se aspectos biopsicossociais do adoecimento. Entretanto, segundo Nogueira (2009), ainda se observa muita resistência a essa transformação e, em alguns casos, a formação médica ainda privilegia aspectos mecanicistas, biologicistas e individualizantes.

Quanto ao paradigma psicossocial, a ANOVA executada também indicou que havia diferença entre as médias dos universitários $(\mathrm{F}(2,475)=6,79, p<0,001)$. $\mathrm{O}$ Teste de Bonferroni demonstrou diferença significativa entre os cursos de Psicologia e de Enfermagem $(p<0,001)$, e entre os cursos de Psicologia e de Medicina $(p<0,001)$, assim, os estudantes que apresentaram maior pontuação no paradigma psicossocial foram os do curso de Psicologia.

Acerca dos cursos de Medicina e Enfermagem, Moretti-Pires e Bueno (2009) afirmam que a formação em tais áreas de conhecimento mantém-se centrada em uma visão reducionista da saúde e da doença. Longe do enfoque totalizador do ser humano, essas áreas de conhecimento se pautam, principalmente, no modelo de ensino de técnicas e no desenvolvimento de competências.

Quanto ao curso de Psicologia, a pesquisa conduzida por Gil e Tardivo (2007), com graduandos dessa área de conhecimento, revelou que a maioria deles considerava aspectos ligados ao contexto social, para a compreensão do adoecimento psíquico. Acerca dessa questão, Dimenstein e Macedo (2012) acrescentam que os desdobramentos do movimento da Reforma Psiquiátrica favoreceram a inserção do psicólogo na saúde pública, e foi a saúde mental o setor público onde a entrada desse profissional foi mais intensa; havendo uma ampliação dos postos de trabalho para essa categoria, nas políticas, nos programas e nos projetos sociais ligados à saúde mental.

Nesse contexto, houve investimentos por parte dos Conselhos Federais de Psicologia, em termos da abertura de cursos de graduação e de sua redefinição político-pedagógica, na busca de atender as demandas da saúde pública, em especial da saúde mental 
(Dimenstein \& Macedo, 2012). Assim, a Psicologia vem buscando uma formação/atuação congruente com a atual configuração do campo da saúde mental, pautada na Reforma Psiquiátrica, visto que tal campo constitui um amplo e crescente mercado de atuação (Souza \& Delevati, 2013). Pode-se concluir, portanto, que a trajetória da Psicologia na saúde mental tem um papel relevante na maior adesão dos seus universitários ao paradigma psicossocial de atenção à saúde mental.

Diante do exposto, pode-se observar que os universitários apresentam algumas diferenças na assimilação dos preceitos da reforma. Sobre isso, Borges e Luzio (2010), a reforma psiquiátrica ainda é um processo em construção e devido a isso a atenção psiquiátrica pautada nos serviços extra-hospitalares convive, lado a lado, com a permanência do paradigma biomédico, centrado na internação em instituições psiquiátricas.

Essa dificuldade de passagem do paradigma biomédico para o psicossocial, para a efetivação da reforma psiquiátrica e da desinstitucionalização, deve-se a vários fatores, dentre os quais cabe ressaltar o despreparo da sociedade para o convívio com a loucura, evidenciado pelo preconceito que os indivíduos mantêm acerca da doença mental (Andrade, Medeiros, \& Patriota, 2010); representações essas refletidas da sociedade para a academia.

\section{Técnica de Associação Livre de Palavras}

A Técnica de Associação Livre de Palavras (TALP) foi utilizada para conhecer e analisar a estrutura da representação social da Reforma Psiquiátrica e do doente mental, elaboradas por estudantes universitários dos cursos de Psicologia, Medicina e Enfermagem. Com o uso dessa técnica, buscou-se compreender, mais profundamente, as diferenças encontradas por meio da Escala de Atitudes em Saúde Mental. A partir da análise realizada pelo EVOC, foram elaboradas as Figuras 1 e 2 , contendo as palavras evocadas, a frequência e a ordem média de evocação.

A Figura 1 mostra os quadrantes relativos à estrutura central e à estrutura periférica da representação social elaborada pelos estudantes dos cursos de Psicologia, Medicina e Enfermagem, acerca do estímulo indutor Reforma Psiquiátrica. Nos quadrantes esquerdos estão as evocações que correspondem ao núcleo central dessa representação. Ao analisar tais evocações, percebe-se que o termo "mudança" está presente nos três cursos. Isso demonstra que, entre esses universitários, existe a compreensão da Reforma Psiquiátrica

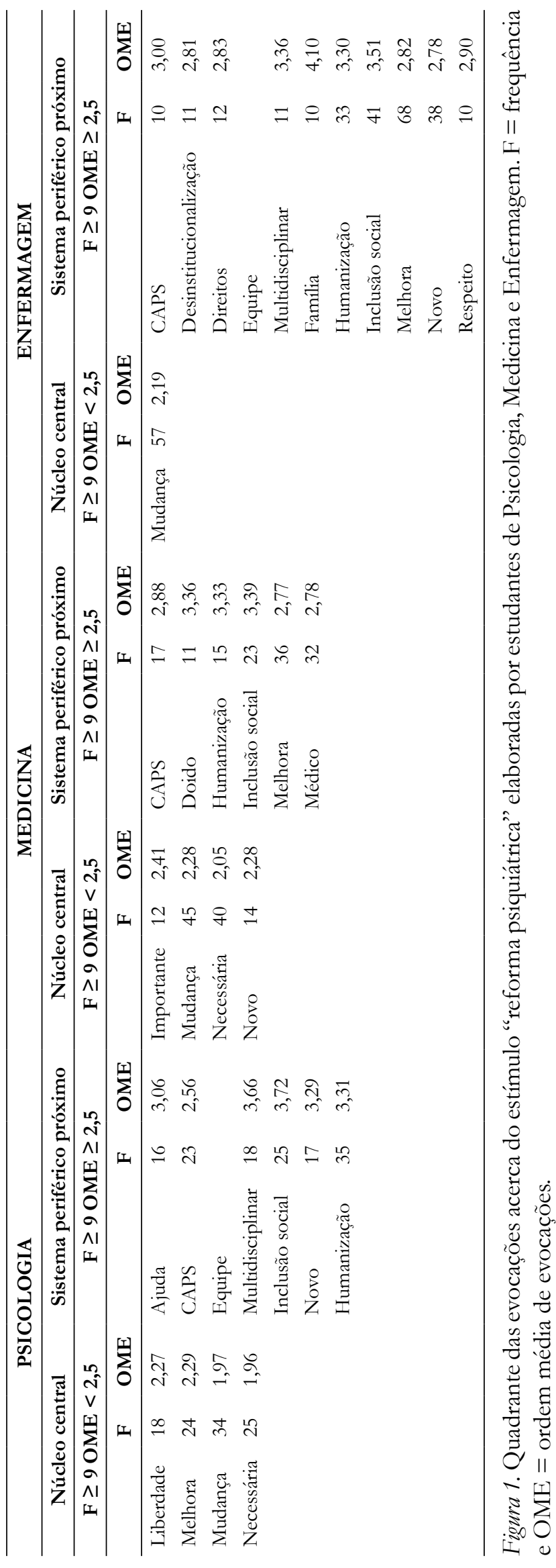

Psico-USF, Bragança Paulista, v. 21, n. 3, p. 527-538, set./dez. 2016 
como algo que propõe transformações na assistência à saúde mental.

Ainda quanto à composição do núcleo central, observa-se, entre os estudantes dos cursos de Psicologia e Medicina, a presença de outras palavras, tais como "melhora, necessária", "importante" e "novo", que exprimem uma compreensão da Reforma enquanto algo positivo e que corresponde à atual política de saúde mental. Verifica-se, também, a evocação "liberdade", fazendo referência à desospitalização.

Calgaro e Souza (2009) assinalam que a atual política de saúde mental, pautada na Reforma Psiquiátrica, propõe alternativas substitutivas ao modelo hospitalocêntrico, incluindo a implantação de uma rede de serviços territoriais de atenção psicossocial, buscando a integração do indivíduo em sofrimento psíquico ao meio social. Portanto, a mudança proposta por essa nova política de saúde mental consiste na transição do paradigma biomédico, centrado na internação em instituições psiquiátricas, para o paradigma psicossocial, baseado nos serviços extra-hospitalares de base comunitária (Costa-Rosa, 2012).

Abric (2003) afirma que o núcleo central é o elemento unificador e estabilizador da representação social. Nesse sentido, as expressões que compõem o núcleo central evidenciam uma representação social semelhante acerca da Reforma Psiquiátrica entre os universitários dos três cursos, demonstrando que há entendimentos acerca das propostas gerais dessa política entre os estudantes dos cursos de Psicologia, Medicina e Enfermagem. Esse fato corrobora os dados encontrados por meio da Escala de Atitudes em Saúde Mental, que indicaram uma atitude pautada no paradigma psicossocial entre os acadêmicos dos três cursos.

No que se refere ao sistema periférico, nota-se, entre os três cursos, a presença de termos que são característicos da assistência à saúde mental pautada na Reforma Psiquiátrica: "CAPS", "inclusão social", "humanização", "família", "direitos", "ajuda" "respeito", "melhora" e "desinstitucionalização". Abric (2003) afirma que os elementos do sistema periférico são responsáveis pela concretização do sistema central, no que se refere às tomadas de posição e de condutas. Dessa forma, orientam as práticas dos estudantes do presente estudo, em congruência com a Reforma Psiquiátrica.

Semelhante aos dados dessa pesquisa, estudo conduzido por Barros e Claro (2011) com estudantes de enfermagem, permitiu identificar entre esses universitários, durante as aulas práticas da disciplina de
Enfermagem em Saúde Mental e Psiquiátrica, atitudes terapêuticas reabilitatórias para com a população assistida, demonstrando conhecimento acerca de uma atuação conforme preconiza a Reforma Psiquiátrica.

Com relação aos elementos periféricos, a expressão "equipe multidisciplinar" foi verificada entre os estudantes dos cursos de Psicologia e de Enfermagem, acentuando a importância do trabalho em equipe; enquanto que os estudantes do curso de Medicina evocaram a palavra "médico", denotando uma compreensão da assistência centralizada na figura do médico, ao invés de na equipe multidisciplinar, uma das características do paradigma psicossocial.

Entre os estudantes de Medicina, verificou-se ainda o termo "doido", que remete a uma representação negativa do doente mental. Observa-se que, apesar de mudanças já estarem acontecendo na sociedade, alguns elementos do sistema periférico evidenciam características do modelo biomédico.

No que se refere às representações elaboradas pelos estudantes dos cursos de Psicologia, Medicina e Enfermagem, acerca do estímulo indutor "doente mental”, observados na Figura 2 abaixo, serão descritos os quadrantes referentes ao sistema central e ao sistema periférico.

Em cada quadrante esquerdo da Figura 2, encontram-se as evocações que correspondem ao núcleo central da representação social acerca do doente mental elaborada pelos universitários. Observa-se que, entre os estudantes dos três cursos, os termos "doença" e "doido" aparecem como elementos constituintes do núcleo central. Entre os estudantes de Medicina surgem, além de tais palavras, as evocações "esquizofrenia" e "problema". Segundo Abric (2003), os elementos do núcleo central são determinados por aspectos históricos e ideológicos, sendo responsáveis pela continuidade, consistência e permanência da representação.

A evocação "doença” faz referência a alguém que precisa de cuidados, assistência, revelando uma concepção de doença mental como vinculada à apropriação do saber médico, que detém o poder de cura. Já a evocação "doido" é usada em nossa cultura como sinônimo de louco e apresenta uma conotação negativa, conforme Maciel, Barros, Camino e Melo, (2007), denotando o pouco valor que a sociedade atribui ao doente mental (Maciel et al., 2011).

Entre os estudantes de Medicina, observou-se, também, no núcleo central, a presença das palavras "esquizofrenia" e "problema". A primeira evocação faz referência à compreensão do indivíduo em sofrimento 


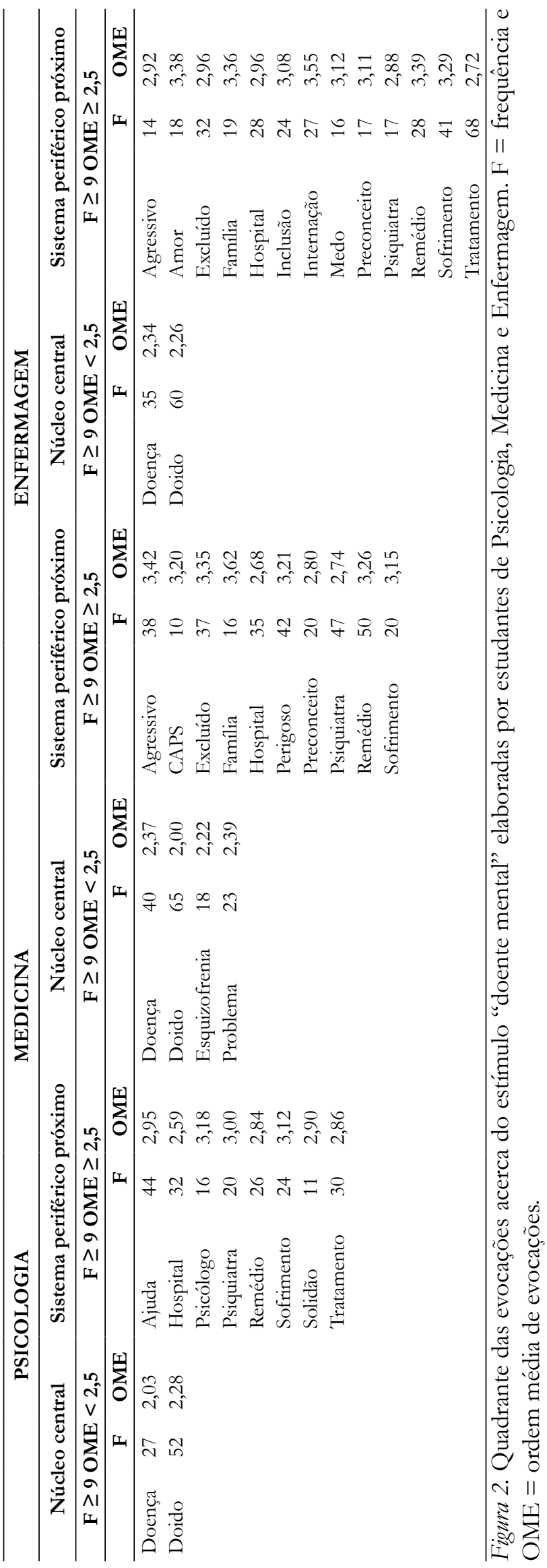

psíquico como portador de uma doença, e não como sujeito; destaca-se aqui a presença do binômio diagnóstico/rotulação, característico do paradigma biomédico. De maneira similar, a segunda palavra "problema" está ligada a uma concepção negativa do doente mental, que ainda se encontra fortemente arraigada na sociedade.

Sobre tais aspectos, Maciel, Maciel, Barros, Sá e Camino (2008) esclarecem que, ainda hoje, o portador de sofrimento psíquico é enxergado de forma negativa pela sociedade, como um indivíduo sem razão, sem juízo e agressivo. Portanto, ele é considerado como perigoso e incapaz de viver em sociedade, precisando ser excluído da família e do meio social e mantido em instituições psiquiátricas, sob a tutela do saber médico. Em suma, os elementos constituintes do núcleo central da representação do doente mental elaborada pelos três cursos remetem a uma representação social perpassada pelo paradigma biomédico e marcada pelo medo e exclusão.

Sobre isso, Abric (2003) ressalta que o núcleo central é o elemento fundamental da representação e que uma representação é suscetível variar por uma mudança no sentido ou na natureza dos seus elementos periféricos, mas ela só se transforma quando o núcleo central é posto em questão.

Quanto aos elementos do sistema periférico próximo, observam-se, entre os três cursos, palavras que demonstram uma compreensão do doente mental atrelada ao paradigma biomédico, tais como "hospital", "psiquiatra", "psicólogo", "remédio", "internação" e "tratamento". O que parece ser um desdobramento dos elementos do núcleo central, reforçando uma compreensão do doente mental atrelada ao modelo ultrapassado de assistência, que tem como base a medicalização, hospitalização e tutela; fato associado à dificuldade de superação do paradigma biomédico, conforme ainda se verifica em nossa sociedade. Observa-se também, entre os três cursos, evocações como "sofrimento", "excluído", "solidão", que parecem remeter a uma compreensão acerca da dificuldade de inserção social vivenciada pelos doentes mentais.

Cabe destacar, ainda, que entre os estudantes dos cursos de Medicina e de Enfermagem, observou-se a presença dos termos "preconceito", "agressivo", "perigoso" e "medo", revelando uma concepção do doente mental vinculada ao estereótipo de periculosidade, de acordo com Sadow e Ryder (2008), este é um dos estereótipos mais comumente associados à doença mental. Sobre isso, pesquisa realizada por Oliveira, Lima, Silva, Oliveira e Alves (2011), com estudantes 
de Enfermagem, também revelou que tais acadêmicos percebiam o doente mental como agressivo e perigoso, com relatos de medo e receio por parte dos estudantes durante a realização dos estágios em saúde mental.

Por fim, entre os estudantes dos três cursos, foram identificadas as palavras "família", "amor", "ajuda", "CAPS" e "inclusão". As palavras "CAPS", "família" e "inclusão" fazem referência a aspectos que caracterizam atuação em congruência com a Reforma Psiquiátrica e as evocações "amor" e "ajuda" realçam sentimentos de acolhimento e humanização, também característicos da atual política de saúde mental.

Diante desses achados, pode-se concluir que esses universitários apresentam uma ambiguidade quanto à absorção de todos os preceitos da Reforma Psiquiátrica, pois por um lado demonstram conhecimentos acerca das práticas que pautam esse novo modelo de assistência à saúde mental, observado por meio da maior pontuação no paradigma psicossocial e da representação social elaborada acerca da Reforma, apresentando elementos que caracterizam essa nova política de saúde, mas apresentam uma representação social do doente mental perpassada pelo medo, pela exclusão e vinculada ao paradigma biomédico. Tal dado parece ser um reflexo da atual situação do campo da saúde mental, na qual o paradigma biomédico não foi totalmente superado e nem o paradigma psicossocial totalmente assimilado, resultando em uma convivência lado a lado dos dois paradigmas, conforme afirmam Borges e Luzio (2010).

Estsa confusão quanto à assimilação dos preceitos da Reforma Psiquiátrica também foi verificada por Castro (2009), entre profissionais de um CAPS e de um manicômio judiciário. Segundo o autor, tais profissionais apresentavam discursos marcados pelo paradigma psicossocial e uma prática característica do paradigma biomédico.

Nesse sentido, percebece-se que apesar das propostas da Reforma Psiquiátrica serem amplamente difundidas e conhecidas entre os universitários, o preconceito frente ao doente mental ainda é muito presente entre esses indivíduos, ancorado em ideias e valores da era manicomial, caracterizada pela exclusão, pela medicalização e pela tutela dos portadores de transtornos mentais. Acerca disso, Maciel (2012) pontua que a ruptura definitiva com o manicômio deve contrapor-se à negatividade patológica, que articula conceitos, como incapacidade, periculosidade, invalidez e inimputabilidade como modos próprios e inerentes à loucura. Assim, é necessário que a Reforma atinja o campo ideológico, transformando a relação da sociedade com a loucura, de forma a combater o preconceito e a exclusão social.

Sobre isso, Pacheco (2011) afirma que se vive hoje uma transição de modelos de atenção à saúde mental, considerando que se busca superar o modelo hospitalocêntrico em vista de um modelo de reabilitação social. Entretanto, mesmo com todo trabalho realizado para consolidação de uma política nacional de saúde mental voltada para a desinstitucionalização, o modelo anterior não foi superado.

\section{Considerações Finais}

Este estudo teve como objetivo conhecer e analisar as representações sociais de estudantes dos cursos de Psicologia, Medicina e Enfermagem sobre a Reforma Psiquiátrica e o doente mental, relacionando-as com a sua adesão aos paradigmas de atenção à saúde mental. Os achados desta pesquisa indicaram, entre os universitários, um conhecimento acerca dos preceitos teóricos que caracterizam a Reforma Psiquiátrica, dado evidenciado pela atitude dos universitários dos três cursos ser pautada no paradigma psicossocial e pelas suas representações acerca da reforma estarem de acordo com o que preconiza a atual política do campo da saúde mental. Contudo, esses estudantes demonstraram uma representação do doente mental marcada pelo medo e exclusão, pautada no paradigma biomédico. No núcleo central das representações sociais do doente mental, onde se concentram os elementos mais rígidos e de mais difícil mudança, observaram-se palavras como "doença" e "doido", demonstrando o quão fortemente a visão estigmatizada do doente mental ainda se faz presente.

Diante de tais aspectos, é coerente assumir que a visão negativa do doente mental, marcada por estereótipos, representa um dos grandes desafios para a inclusão do portador de transtorno mental e para a efetivação da Reforma Psiquiátrica, demandando tempo, dedicação e persistência para a sua superação, até mesmo entre os futuros profissionais da rede de atenção à saúde mental.

A análise das atitudes e representações sociais de universitários sobre a Reforma Psiquiátrica e o doente mental são de grande importância para a compreensão do comportamento desses futuros profissionais do campo da saúde mental, permitindo identificar como esses universitários compreendem as mudanças ocorridas no âmbito da saúde mental, em decorrência da Reforma Psiquiátrica, e em quais aspectos teóricos estarão pautadas suas futuras práticas. 
Assim, pode-se afirmar que a real inclusão do portador de transtorno mental no meio social é um processo bastante complexo e que, apesar de muitas mudanças, ainda há muito o que melhorar, pois não é unicamente a convivência com o doente mental na sociedade o que se busca, mas sim a convivência desprovida de todo o preconceito que existe em torno dos transtornos mentais. Sabe-se que a conquista desse ideal passa, necessariamente, por várias instâncias: legislativa, política, administrativa e de organização de serviços. Entretanto, cabe ressaltar que a desmistificação da doença mental precisa ser pensada não somente em termos macroestruturais, mas em ações e mudanças no plano microestrutural.

Cabe acrescentar que essa pesquisa apresenta como limitação a impossibilidade de generalização dos resultados para outras amostras, uma vez que esses achados fazem referência a um grupo específico de universitários, localizados em um contexto específico. Contudo, os resultados ora apresentados não perdem sua relevância em termos de conhecimento dessa realidade e da possibilidade de refletir sobre essas questões com vistas a avanços no campo da inclusão social e de políticas públicas mais humanitárias.

Ressalta-se, por fim, a importância de novos estudos acerca do doente mental, do preconceito e do processo de inclusão social, envolvendo outros setores da sociedade, como os profissionais de saúde, familiares e a população em geral, uma vez que a efetivação do processo de inclusão social requer a contribuição dos vários setores da sociedade, os quais devem atuar no sentido de aceitar e acolher o doente mental em seu cotidiano. De forma que, os resultados alcançados possam servir de embasamento para a proposição de políticas públicas que favoreçam a efetivação do paradigma psicossocial na sociedade, enfatizando a desconstrução de representações negativas e o incentivo de atitudes mais positivas e inclusivas.

\section{Referências}

Abric, J. C. (2003). L'analyse structurale des représentations sociales. Em S. Moscovici, \& F. Buschini (Eds.), Les méthodes des sciences humaines (pp. 375392). Paris: Presses Universitaires de France.

Andrade, M. T. M., Medeiros, E. F., \& Patriota, L. M. (2010). A reforma psiquiátrica na prática e a prática da reforma psiquiátrica: Um estudo a partir das percepções dos profissionais do CAPSI (centro campinense de intervenção precoce) de Campina
Grande - PB. Cadernos Brasileiros de Saúde Mental, 2(4), 47-59.

Barros, S., \& Claro, H. G. (2011). Processo ensino-aprendizagem em saúde mental: $\mathrm{O}$ olhar do aluno sobre reabilitação psicossocial e cidadania. Revista da Escola de Enfermagem da USP, 45(3), 700-707. doi: 10.1590/S0080-62342011000300022

Borges, R. F., \& Luzio, C. A. (2010). Pesquisa qualitativa em saúde mental: Alguns apontamentos. Revista de Psicologia da UNESP, 9(1), 14-23.

Calgaro, A., \& Souza, E. N. (2009). Percepção do enfermeiro acerca da prática assistencial nos serviços públicos extra-hospitalares de saúde mental. Revista Gaúcha de Enfermagem, 30(3), 476-483.

Castro, U. R. (2009). Reforma Psiquiatrica e o louco infrator: Novas ideias e velhas práticas (Dissertação de mestrado). Universidade Católica de Goiás, Goiânia, Goiás.

Costa-Rosa, A. (2012). A instituição de saúde mental como dispositivo social de produção de subjetividade. Estudos de Psicologia, 29(1), 115-126. doi: 10.1590/S0103-166X2012000100013

Dimenstein, M., \& Macedo, J. P. (2012). Formação em psicologia: Requisitos para atuação na atenção primária e psicossocial. Revista Psicologia Ciência e Profissão, 32(número especial), 232-245. doi: 10.1590/ S1414-98932012000500017

Fagundes, M. M., Zanella, M., \& Torres, T. L. (2012). Cidadão em foco: Representações sociais, atitudes e comportamentos de cidadania. Psicologia: Teoria e Prática, 14(1), 55-69.

Field, A. (2009). Descobrindo a estatística usando o SPSS. Porto Alegre: Artmed.

Foucault, M (2012). História da loucura na idade clássica $\left(1^{\mathrm{a}}\right.$ Reimpressão). São Paulo: Perspectiva.

Gil, C. A., \& Tardivo, L. C. (2007). Concepção de doença mental em estudantes de graduação em psicologia: Um estudo compreensivo por meio de desenhos temáticos. Mudanças em Psicologia da Saúde, 15(2), 114-120.

Gonçalves, M. B., \& Benevides-Pereira, A. M. T. (2009). Considerações sobre o ensino médico no Brasil: Consequências afetivo-emocionais nos estudantes. Revista Brasileira de Educaşão Médica, 33(3), 493-504. doi: 10.1590/S0100-55022009000300020 
Guimarães, A. N., Fogaça, M. M., Borba, L. O., Paes, M. R., Larocca, L. M., \& Maftum, M. A. (2010). O tratamento ao portador de transtorno mental: Um diálogo com a legislação federal brasileira (1935-2001). Texto \& Contexto-Enfermagem, 19(2), 274-282. doi: 10.1590/S0104-07072010000200008

Jodelet, D. (2001). Representações sociais: um domínio em expansão. Em D. Jodelet (Eds.), As representações sociais (pp.17-41). Rio de Janeiro: Ed UERJ.

Liberato, M (2009). Desinstitucionalizar é ultrapassar fronteiras sanitárias, o desejo da intersetorialidade do trabalho em rede. Cadernos Brasileiros de Saúde Mental, 1(1), 1-10.

Lima, M. L. (2006). Atitudes: Estrutura e mudança. Em J. Vala \& M. Jablonski (Eds.), Psicologia social (pp. 187-225). Lisboa: Fundação Calouste Gulbenkian.

Maciel, S. C. (2007). Exclusão/inclusão social do doente mental/ louco: Representações e práticas no contexto da reforma psiquiátrica (Tese de doutorado). Universidade Federal da Paraíba, João Pessoa, Paraíba.

Maciel, S. C. (2012). Reforma psiquiátrica no Brasil: algumas reflexões. Cadernos Brasileiros de Saúde Mental, 4(8), 73-82.

Maciel, S. C., Barros, D. R., Camino, L. F., \& Melo, J. R. F. (2011). Representações sociais de familiares acerca da loucura e do hospital psiquiátrico. Temas em Psicologia, 19(1), 193-204.

Maciel, S. C., Maciel, C. M. C., Barros, D. R., Sá, R. C. N., \& Camino, L. F. (2008). Exclusão social do doente mental: Discursos e representações no contexto da reforma psiquiátrica. Psico-USF, 13(1), 115-124.

Menegat, D. R. (2010). A desinstitucionalização do portador de doença mental e a reforma psiquiátrica brasileira. Revista do Ministério Público do RS, 66(1), 33-64.

Moretti-Pires, R. O., \& Bueno, S. M. V. (2009). Freire e formação para o Sistema Único de Saúde: $\mathrm{O}$ enfermeiro, o médico e o odontólogo. Acta Paulista de Enfermagem, 22(4), 439-444.

Moscovici, S. (1961). La psichologie, son image et son publique. Paris: PUF.

Nóbrega, S. M., \& Coutinho, M. P. L. (2011). O Teste de associação livre de palavras. Em M. P. L. Coutinho \& E. R. A. Saraiva (Eds.), Métodos de pesquisa em
Psicologia Social, perspectivas qualitativas e quantitativas (pp. 95-106). João Pessoa: Editora Universitária.

Nogueira, M. I. (2009). As mudanças na educação médica brasileira em perspectiva: Reflexões sobre a emergência de um novo estilo de pensamento. Revista Brasileira de Educação Médica, 33(2), 262-270. doi: 10.1590/S0100-55022009000200014

Oliveira, R. M., Lima, A. E. S., Silva, D. G., Oliveira, M. D. F., \& Alves, K. S. M. (2011). Visão dos acadêmicos de enfermagem em relação ao cliente portador de transtorno mental. Revista Cadernos de Ciência e Saúde, 1(2), 75-85.

Pacheco, J. G. (2011). Representacoôes sociais da loucura e práticas sociais: $O$ desafio cotidiano da desinstitucionalização (Tese de doutorado). Universidade de Brasília, Brasília, Distrito Federal.

Paes, M. R., Maftum, M. A., \& Mantovani, M. F. (2010). Cuidado de enfermagem ao paciente com comorbidade clínico-psiquiátrica em um pronto atendimento hospitalar. Revista Gaúcha de Enfermagem, 31(2), 277-284. doi: 10.1590/ S1983-14472010000200011

Pagliosa, F. L. (2006). Percepcõos sobre saúde e doença dos estudantes da primeira fase do curso de medicina da UFSC (Dissertação de mestrado). Universidade Federal de Santa Catarina, Florianópolis, Santa Catarina.

Parente, A. C. M., Menezes, L. C., Branco, F. M. F. C., Sales, J. C. S., \& Parente, A. C. B. V. (2013). Reforma da assistência psiquiátrica brasileira: Realidade e perspectiva. Revista de Enfermagem do UFPI, 2(2), 66-73.

Sá, C. P. (1996). Sobre o núcleo central das representações sociais. Petrópolis: Vozes.

Sadow, D., \& Ryder, M. (2008). Reducing stigmatization attitudes held by future health professionals: The person is the message. Psychological Services, 5(4), 362-372.

Saraiva, E. R. A., \& Coutinho, M. P. L. (2007). A estrutura das representações sociais de mães puérperas acerca da depressão pós-parto. Psico-USF, 12(2), 319-326.

Saraiva, E. R. A., Vieira, K. F. L., \& Coutinho, M. P. L. (2011). A utilização do software EVOC nos estudos acerca das representações sociais. Em M. P. L. Coutinho \& E. R. A. Saraiva (Eds.), Métodos de pesquisa em Psicologia Social, perspectivas qualitativas 
e quantitativas (pp. 149-174). João Pessoa: Editora Universitária.

Souza, A. R. B., \& Delevati, D. (2013). O fazer do psicólogo na saúde. Cadernos de Graduação - Ciências Biológicas e da Saúde Fits, 1(2), 79-87.

Sousa, P. F., Maciel, S. C., Torres, A. R. R., Lima, T. J. S., Medeiros, K. T., \& Silva, G. L. S. (no prelo). Evidências de validade fatorial e precisão da escala de atitudes em saúde mental. Estudos de Psicologia.
Vieira, K. F. L., Saraiva, E. R. A., \& Coutinho, M. P. L. (2010). Entrelaçamentos entre depressão e suicídio segundo os futuros psicólogos. Revista Psico, 41(2), 176-183.

Recebido 16/02/2015

Reformulado 09/09/2015

Aceito 06/10/2015

Sobre as autoras:

Patrícia Fonseca de Sousa é psicóloga, mestre, doutoranda em Psicologia Social pela Universidade Federal da Paraíba e pesquisadora do Grupo de Pesquisa em Saúde Mental e Dependência Química (UFPB).

E-mail: patriciasousa20@yahoo.com.br

Silvana Carneiro Maciel é doutora em Psicologia pela Universidade Federal da Paraíba e pela Universidade Federal do Rio Grande do Norte (UFPB/UFRN), com pós-doutorado pelo Instituto de Ciências Sociais da Universidade de Lisboa (ICS-Lisboa), professora do Departamento de Psicologia e do Programa de Pós-graduação em Psicologia Social (UFPB) e coordenadora do Grupo de Pesquisa em Saúde Mental e Dependência Química (UFPB).

E-mail: silcamaciel@gmail.com

Katruccy Tenório Medeiros é psicóloga, mestre e doutoranda em Psicologia Social pela Universidade Federal da Paraíba e pesquisadora do Grupo de Pesquisa em Saúde Mental e Dependência Química (UFPB).

E-mail: katruccy_22@yahoo.com.br

Giselli Lucy Souza Vieira é psicóloga, especialista em Saúde Mental pelas Faculdades Integradas de Patos (FIP), mestre, doutoranda em Psicologia Social pela Universidade Federal da Paraíba e pesquisadora do Grupo de Pesquisa em Saúde Mental e Dependência Química (UFPB).

E-mail: giselli_psi@hotmail.com

Contato com as autoras:

Rua Vinícius barbalho, 82, Cohab

CEP: 59173-000

Goianinha-RN, Brasil

E-mail: patriciasousa20@yahoo.com.br 